\section{Patient communication during cataract surgery}

A Mokashi, B Leatherbarrow, J Kincey,

R Slater, $\mathrm{V}$ Hillier and S Mayer

\begin{abstract}
Purpose Most cataract surgery is now performed under local anaesthesia on a daycase basis. As patients are fully conscious during the procedure, it is important that they remain still. There are a variety of reasons why patients may need to move, and it is important that the surgeon is made aware that this may happen. Some centres offer a nurse's hand as a means of perioperative patient communication. We sought to study the safety and efficacy of using an electronic patient-controlled alert device.

Method We compared hand-holding with the use of a patient alert device, and with both communication methods at the same time, on 150 subjects undergoing cataract surgery under local anaesthesia. Assessment of pre- and postoperative state anxiety was undertaken and patients' satisfaction with the communication strategies was assessed. Results There was a significant difference between pre- and postoperative state anxiety for each group $(P<0.001)$ but no significant differences in pre-, peri-, or postoperative state anxiety between groups. There were no significant differences in confidence, pain, understanding, satisfaction, memory, and reassurance between the three groups. A total of $46 \%$ of all patients reported experiencing one or more of the potential problems enquired about, during the operation. Significant correlations were also identified between some of the psychological variables investigated.

Conclusions An electronic patient alert device is as effective a means of perioperative patient communication as holding a nurse's hand, during cataract surgery under local anaesthesia. It is safe, reassuring and it allows patients to communicate directly with the surgeon.

Eye (2004) 18, 147-151. doi:10.1038/

sj.eye. 6700568
\end{abstract}

Keywords: cataract; local anaesthesia

\section{Introduction}

Patients undergoing cataract surgery under local anaesthesia are required to remain immobile, supine, and have their faces and upper body covered. Many have coexisting health complaints. ${ }^{1}$ Patients with cardiorespiratory disease, those with continence problems, those with generalised arthritis, and those who are anxious may find it difficult to relax and remain still during the operation. It is important to take into account patient preference, anxiety, and ability to cooperate, and to assure patients that they will be carefully monitored. ${ }^{2}$

Patients should have an effective means of communicating any problems that they experience during the procedure to the surgeon. Vocalising any concerns may cause facial or head movements, which would be undesirable during an intraocular procedure. It seems logical to assume that providing such a means would lead to greater patient satisfaction and may reduce the risk of perioperative complications.

Surgery should be viewed as both a physical procedure and as an experience with a unique meaning for the individual patient. Patients need an appropriate understanding of the surgical procedure. Failures of understanding have been consistently demonstrated within other aspects of healthcare and treatment, and there are important and significant relations between the levels of understanding and memory for medical information, the levels of satisfaction with communication from clinicians, and the levels of anxiety experienced by patients. ${ }^{3,4}$ There is a risk that, in the drive to increase surgical throughput, the psychological welfare of the surgical patient may be reduced in importance. ${ }^{5}$

In our unit, patients are given a trained nurse's hand to hold during their operation. The patient is instructed to squeeze the nurse's hand in the event of any problems. Hand-holding as a
Manchester Royal Eye Hospital

Manchester, UK

Correspondence:

B Leatherbarrow

Manchester Royal Eye

Hospital

Oxford Road

Manchester M13 9WH, UK

Tel: + 441612765569

Fax: + 441612726618

E-mail: bollin@

mighty-micro.co.uk

Received: 4 November 2003 Accepted in revised form: 24 January 2003 
means of perioperative communication, however, it is not without its flaws. Anxious patients tend to squeeze the nurse's hand throughout the procedure, making it difficult for the nurse to determine whether or not the patient has a problem. Some patients may find it uncomfortable holding a stranger's hand. Most importantly, there is no direct means of communication with the surgeon. In addition, the trained nursing staff could be used more efficiently performing other intraoperative tasks, although the human contact conveyed through hand-holding can be reassuring and comforting.

A possible alternative to hand-holding is the use of a patient-controlled audible alert device (PAD). Such a device should ideally provide patients with an effective means of communicating any problems experienced perioperatively to the surgeon promptly and directly, and be safe, comfortable, and easy to use.

We report the results of a controlled clinical trial investigating the safety and efficacy of the use of a PAD as a means of patient communication during cataract surgery performed under local anaesthesia.

\section{Materials and methods}

After obtaining Ethics Committee approval, informed consent was sought from 168 patients attending the Manchester Royal Eye Hospital, for first eye cataract extraction under local anaesthesia, for inclusion in the study, after which 160 patients agreed to take part. The age range was 48-91 years. These patients were randomly allocated before the start of surgery to receive either the hand-holding protocol, the PAD, or both concurrently.

The PAD was designed bearing in mind the following criteria:

- a patient activated audible alarm,

- an alarm sound sufficiently unique to avoid confusion with other theatre equipment,

- ease of activation, particularly for patients with arthritis/upper limb weakness,

- patient safety and comfort.

The resulting device (Figure 1) was a switch-activated audible alarm which was pulsed on and off at $50 \mathrm{~Hz}$ to produce a distinctive sound at a level of $85 \mathrm{~dB}$. The alarm was activated by an ultra-low-pressure switch connected to an air actuator moulded in black PVC. The unit was operated from a single $9 \mathrm{~V} \mathrm{pp} 3$ battery.

Preoperatively patients were informed of the need to remain still during the operation but were instructed on the methods of communicating problems to the surgeon (squeezing the nurse's hand, the PAD, or both, depending on which had been allocated). The patients

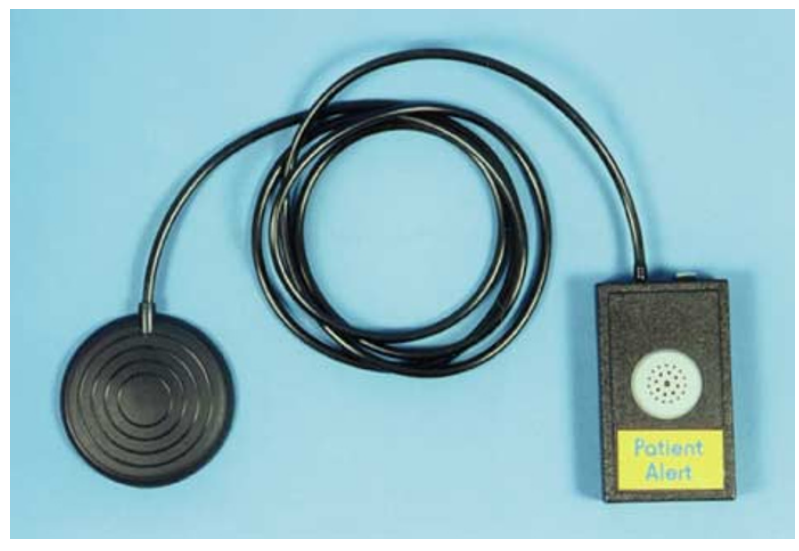

Figure 1 Patient-controlled audible alert device (PAD).

were reassured that the surgeon would then stop as soon as was safely possible and enquire about their problem. Patients were given the opportunity to familiarise themselves with the PAD before the start of surgery.

Local anaesthesia was administered by means of either a sub-Tenon's block (90 cases), a peribulbar block (53), or topically (7). Patients were given supplemental oxygen $41 /$ min via an open box under the drapes. Pulse oximetry and ECG were monitored continuously (Datex AS3). The duration of operation recorded was that from the placement of the drape to its removal.

The following psychological parameters were measured:

1. Anxiety assessment: Six-item short form of the state scale of the Spielberger State-Trait Anxiety Inventory (STAI), $1 \mathrm{~h}$ preoperatively and $1 \mathrm{~h}$ postoperatively. ${ }^{6}$

2. Confidence and reassurance in using allocated communication method, pain, understanding of procedure, perioperative anxiety, satisfaction with information about the operation, and memory of what happened during the operation. This was assessed by a specifically constructed self-evaluation questionnaire $1 \mathrm{~h}$ postoperatively (Table 1 ).

Data regarding the types of problems patients undergoing cataract extraction under local anaesthesia may encounter were also collected, as were data regarding the use of the allocated perioperative communication method (Table 1).

\section{Data analysis}

Grouping was as follows: group 1 -hand-holding; group 2-PAD; group 3-hand-holding and PAD. Statistical analysis of data was carried out using nonparametric tests. Data for duration, preoperative, postoperative, and self-evaluation questionnaires were analysed. A $P$ value 
Table 1 Self-evaluation questionnaire

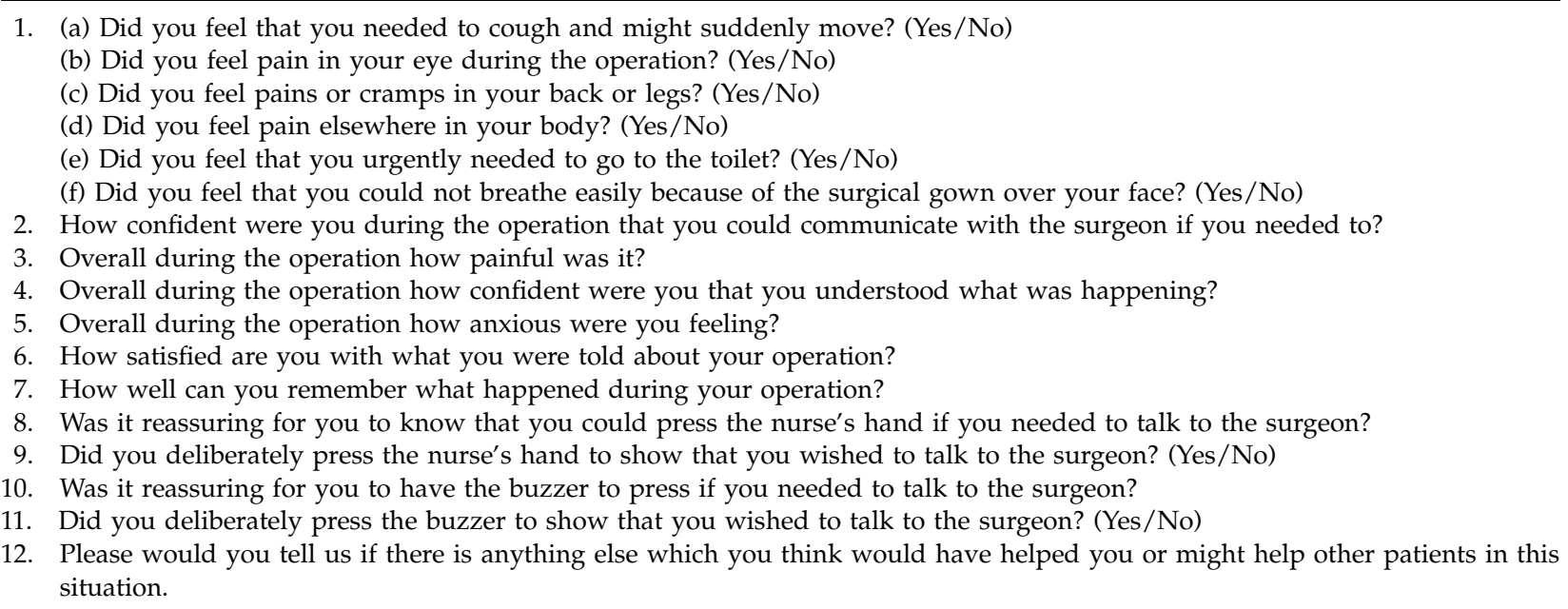

Scores: not at all=1, somewhat=2, moderately $=3$, completely/very much $=4$ (with reversed scoring where appropriate, high scores indicating a positive outcome, for example, low pain or less anxiety). Questions 1, 9 and 11: Yes $=1, \mathrm{No}=0$.

Table 2 Mean (median) age, duration of operation and pre- and postoperative stateanxiety mean scores

\begin{tabular}{llccc}
\hline Group & Age (years) & Duration (min) & Preoperative state anxiety & Postoperative state anxiety \\
\hline $1(n=50)$ & $76.08(75.50)$ & $23.88(20.50)$ & 10.28 & $7.46^{*}$ \\
$2(n=50)$ & $74.02(75.00)$ & $25.52(23.00)$ & 9.84 & $7.36^{*}$ \\
$3(n=50)$ & $72.62(75.00)$ & $23.40(20.00)$ & 10.04 & $7.74^{*}$
\end{tabular}

*Wilcoxon signed ranks test, $P<0.001$.

less than 0.05 was considered to be statistically significant.

\section{Results}

Of the 168 patients approached to participate in the study, eight patients declined and eight observations were discarded owing to incomplete data. In one case, the patient requested a nurse's hand during surgery, and in a further case it was felt that the PAD alone was not appropriate. This left 150 participants. No episodes of hypoxia (oxygen saturation below 90\%) were observed, nor were there any other cardiorespiratory complications noted. Table 2 shows the mean age and duration of operation, as well as the pre- and postoperative stateanxiety mean scores.

No significant differences existed between the mean age or duration of operation recorded in each group.

The STAI revealed a significant reduction in the mean overall postoperative scores compared with the mean preoperative scores across all three groups, together and separately. There was however no significant difference between the postoperative or preoperative scores recorded in each group.
The self-evaluation questionnaire revealed that of the 150 subjects, 69 (19 from group 1, 23 from group 2, and 27 from group 3) replied yes to one or more of the items of question 1 (Figure 2).

The self-evaluation questionnaire did not reveal any significant differences in the mean scores for questions $2-7$ between the three groups. Figure 3 shows the selfevaluation questionnaire mean scores for questions 2-7 by group.

Across all three groups, most patients found the procedure not at all or just somewhat painful and felt not at all or just somewhat anxious during the procedure, not at all being the response in the majority of cases for both these items. This indicates that most patients were moderately or completely (in the majority of cases) confident, perioperatively, that they could communicate with the surgeon if they needed to, and that they understood what was happening, the same being true for how satisfied they felt with what they were told about their operation and how well they could remember what happened during their operation.

All the correlations revealed between items of the selfevaluation questionnaire were positive, as a high score on the self-evaluation questionnaire indicated a positive 


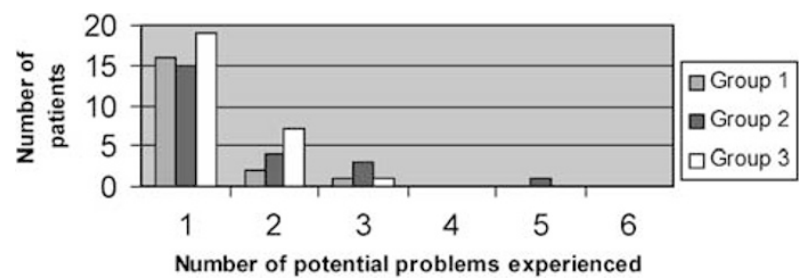

Figure 2 Number of patients in each group who experienced different numbers of potential perioperative problems.

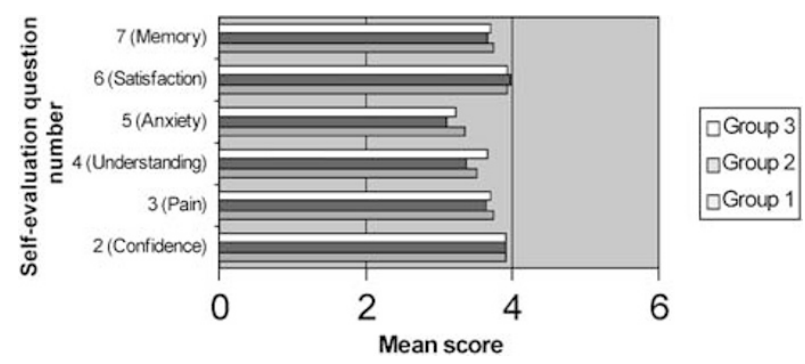

Figure 3 Self-evaluation questionnaire mean scores for each group.

outcome (ie greater understanding, less pain, or less anxiety). A significant correlation was revealed between how confident the patients were that they could communicate with the surgeon during the operation if they needed to and how pain-free the operation was overall $(r=0.179, P=0.024)$, how confident they were that they understood what was happening during the operation $(r=0.257, P=0.001)$, and also how satisfied patients were with what they were told about their operation $(r=0.210, P=0.010)$. A significant correlation was revealed between overall perioperative anxiety and pain $(r=0.159, P=0.035)$. In addition, a just significant correlation was also revealed between perioperative anxiety and the degree to which patients remembered what happened during their operation $(r=0.148$, $P=0.050$ ).

The questionnaire revealed a significant difference (Mann-Whitney $U$ test, $P=0.006$ ) in mean perioperative anxiety scores (question 5) between patients who did (3.30) and did not (2.65) feel able to breathe easily during the operation. A significant difference (Mann-Whitney $U$ test, $P=0.010$ ) in mean perioperative anxiety scores was also revealed between patients who did (2.74) and did not (3.33) feel pain in their eye during the operation. No significant differences were noted in mean perioperative anxiety scores for any of the other items of question 1 of the self-evaluation questionnaire.

The self-evaluation questionnaire revealed that the percentage of patients completely reassured with the allocated means of perioperative communication was $94 \%$ (group 1), 96\% (group 2), and 96\% (group 3).

In addition, no significant differences in type of anaesthetic block used, anaesthetic agent used, or sex of patients was found between the three groups.

\section{Discussion}

Although it would have been ideal to include in the study a group that received no means of perioperative communication, this would have been unethical in the centre in which the study was undertaken, as handholding was offered as standard treatment to patients undergoing cataract surgery. It is therefore not possible to conclude that the observed shift in STAI scores across all three groups was entirely as a result of a means of perioperative communication being offered to the patients. It seems likely that the successful completion of the surgery itself would have had a significant anxiolytic effect on the patients. The fact that there was no significant difference in mean STAI scores between the groups suggests that the three methods of perioperative communication offered were equally effective at providing anxiolysis. It is likely that each method did provide some perioperative anxiolysis, as most patients felt not at all or just somewhat anxious during their operations. Also, the fact that the majority of patients in each group found it completely reassuring to have their allocated means of perioperative communication and were completely confident that they could communicate with surgeon perioperatively suggests that provision of such means did contribute to perioperative anxiolysis.

The frequency with which and the number of different patients for whom the various potential problems occurred suggests that many patients do experience a problem during cataract surgery. However, results also suggest that patients who are more anxious perioperatively are more likely to experience problems. Patients who felt they could not breathe easily and those who felt pain in their eye were more anxious during the operation.

The results indicated no differences in confidence to communicate, overall pain, understanding of the procedure, perioperative anxiety, satisfaction with what was told, and memory, between the three groups. This suggests that neither of the communication means being investigated were 'better' for patients with respect to each of the psychological parameters mentioned above. The study did however reveal interesting relations between some of these parameters. The results showed significant positive correlations between confidence to communicate, understanding of the procedure, satisfaction with what was told about the operation, and overall pain; perioperative anxiety and overall pain; and 
perioperative anxiety and memory of what happened during the procedure. These results suggest that greater understanding leads to greater confidence to communicate, less overall pain, and greater satisfaction with what is told about the operation. They also suggest that less perioperative anxiety leads to less overall pain, or vice versa, and a better memory of what happened during the procedure.

The importance of communication that the clinician provides to patients regarding the procedure is clearly highlighted above. Good communication and information can lead to better patient understanding of the procedure, greater patient confidence, less anxiety, less pain, and greater patient satisfaction, thus potentially leading to a better operative outcome.

Of those patients who had both hand-holding and the PAD during their operation who indicated that they needed to talk to the surgeon, it is interesting to note that the majority used the PAD as opposed to squeezing the nurse's hand. Further research to clarify patient's preferences and expectations would almost certainly be useful.

\section{Conclusions}

Patients undergoing cataract surgery under local anaesthesia can experience a variety of problems during the procedure and need a means of expressing any concerns they may have perioperatively. This study found an electronic PAD to be statistically just as effective a means of perioperative patient communication as holding a nurse's hand. It is safe, it is likely that it provides effective perioperative anxiolysis, patients are confident in using it, and it provides them with reassurance during the operation. It allows patients to communicate promptly and directly with the surgeon rather than via a nurse. In addition, it is much more costeffective a means of perioperative communication than holding a trained nurse's hand. However, not all patients will be suitable to use the patient alert, such as patients suffering with mild dementia. In such cases, it is possible that the human contact conveyed through hand-holding would be particularly reassuring and anxiolytic. Also, patients who are suitable to use the PAD but feel the need for the comfort and reassurance provided by holding a nurse's hand should not be denied it. Therefore, we conclude that patients should be assessed individually for their suitability to use a patient alert, as well as their expected preferences for particular communication strategies. Furthermore, good communication from the clinician about the procedure is more likely to lead to a better patient response to all aspects of the surgery.

\section{References}

1 Lowe K, Gregory D, Jeffery R, Easty D. Cataract extraction: patient characteristics and preferences. Health Trends 1992; 24(4): 151-153.

2 The Royal College of Anaesthetists and the Royal College of Ophthalmologists. Local anaesthesia for intraocular surgery. 2001: 15.

3 Ley P. Communicating with Patients: Improving Communication, Satisfaction and Compliance. Croom Helm: London, 1988.

4 Ley P, Llewelyn S. Improving patients' understanding, recall, satisfaction and compliance. In: Brooke A, Llewelyn S (eds). Health Psychology: Processes and Applications, 2nd edn. Chapman \& Hall: London, 1995, pp 75-98.

5 Kincey J. Surgery. In: Brooke A, Llewelyn S (eds). Health Psychology: Processes and Applications, 2nd edn. Chapman \& Hall: London, 1995, pp 391-403.

6 Marteau TM, Bekker H. The development of a six-item shortform of the state scale of the Spielberger State-Trait Anxiety Inventory (STAI). British Journal of Clinical Psychology 1992; 31: 301-306. 